\title{
A compact linear Paul trap cooler buncher for CRIS
}

DOI:

10.1016/j.nimb.2019.04.054

\section{Document Version}

Accepted author manuscript

Link to publication record in Manchester Research Explorer

\section{Citation for published version (APA):}

Ricketts, C., Cooper, B., Edwards, G., Perrett, H., Billowes, J., Binnersley, C., Cocolios, T., Flanagan, K., Garcia Ruiz, R. F., de Groote, R. P., Gustafsson, F. P., Koszorús, A., Neyens, G., Vernon, A., \& Yang, X. F. (2019). A compact linear Paul trap cooler buncher for CRIS. Nuclear Instruments \& Methods in Physics Research. Section B: Beam Interactions with Materials and Atoms. https://doi.org/10.1016/j.nimb.2019.04.054

\section{Published in:}

Nuclear Instruments \& Methods in Physics Research. Section B: Beam Interactions with Materials and Atoms

\section{Citing this paper}

Please note that where the full-text provided on Manchester Research Explorer is the Author Accepted Manuscript or Proof version this may differ from the final Published version. If citing, it is advised that you check and use the publisher's definitive version.

\section{General rights}

Copyright and moral rights for the publications made accessible in the Research Explorer are retained by the authors and/or other copyright owners and it is a condition of accessing publications that users recognise and abide by the legal requirements associated with these rights.

\section{Takedown policy}

If you believe that this document breaches copyright please refer to the University of Manchester's Takedown Procedures [http://man.ac.uk/04Y6Bo] or contact uml.scholarlycommunications@manchester.ac.uk providing relevant details, so we can investigate your claim.

\section{OPEN ACCESS}




\title{
A compact linear Paul trap cooler buncher for CRIS
}

\author{
C.M. Ricketts ${ }^{\mathrm{a}, *}$, B.S. Cooper ${ }^{\mathrm{a}, \mathrm{b}}$, G. Edwards ${ }^{\mathrm{a}, \mathrm{b}}$, H.A. Perrett ${ }^{\mathrm{a}, \mathrm{b}}$, J. Billowes ${ }^{\mathrm{a}}$, C.L. Binnersley ${ }^{\mathrm{a}}$, T.E. Cocolios ${ }^{\mathrm{c}}$, K.T. Flanagan ${ }^{\mathrm{a}, \mathrm{b}}$, \\ R.F. Garcia Ruiz ${ }^{\mathrm{d}}$, R.P. de Groote ${ }^{\mathrm{f}}$, F.P. Gustafsson ${ }^{\mathrm{c}}$, Á. Koszorús $^{\mathrm{c}}$, G. Neyens $^{\mathrm{c}, \mathrm{d}}$, A.R. Vernon ${ }^{\mathrm{a}}$, X.F. Yang ${ }^{\mathrm{e}}$ \\ ${ }^{a}$ School of Physics and Astronomy, The University of Manchester, Manchester, M13 9PL, United Kingdom \\ ${ }^{b}$ Photon Science Institute, Alan Turing Building, The University of Manchester, Manchester, M13 9PY, United Kingdom \\ ${ }^{c}$ KU Leuven, Instituut voor Kern- en Stralingsfysica, B-3001 Leuven, Belgium \\ ${ }^{d}$ EP Department, CERN, CH-1211 Geneva 23, Switzerland \\ ${ }^{e}$ School of Physics and State Key Laboratory of Nuclear Physics and Technology, Peking University, Beijing 100871, China \\ ${ }^{f}$ Department of Physics, University of Jyväskylä, PB 35 (YFL) FIN-40351 Jyväskylä, Finland
}

\begin{abstract}
A gas-filled linear Paul trap for the Collinear Resonance Ionisation Spectroscopy (CRIS) experiment at ISOLDE, CERN is currently under development. The trap is designed to accept beam from both ISOLDE target stations and the CRIS stable ion source. The motivation for the project along with the current design, simulations and future plans, will be outlined.
\end{abstract}

Keywords: Ion trapping, Laser spectroscopy, 3D printing

\section{Introduction}

Gas-filled linear Paul traps are used at a variety of radioactive beam facilities to provide bunched beams with low emittance to a range of experimental setups [1, 2, 3]. Bunched beams are essential to Collinear Resonance Ionisation Spectroscopy (CRIS) $[4,5,6]$ because the required high-power lasers are only available with repetition rates of the order of $100 \mathrm{~Hz}$, meaning the ion beam must also be pulsed to remove duty-cycle losses. The pulsed-time structure also reduces optical pumping to atomic dark states, further increasing ionisation efficiency [7]. Minimising the ion beam energy spread via in-trap buffergas cooling reduces the Doppler broadening of the resonant excitation steps, leading to higher precision in extracted hyperfine structure and isotope shifts. For more information on the CRIS technique, see the contributions from Á. Koszorús et al. and A.R. Vernon et al. in these proceedings [8, 9].

CRIS at ISOLDE [10] currently utilises the ISCOOL gasfilled linear Paul trap (RFQcb). ISCOOL has been essential to the success of the CRIS experiment, providing bunched beams with $<1 \mathrm{eV}$ energy spread and $<5 \mu$ s width. When fully optimised the transmission efficiency of ISCOOL can reach $70 \%$, however for lower masses the usable efficiency decreases significantly [11]. This article describes the development of an independent RFQcb for the CRIS beamline, to be placed after the CRIS ion source (CRISIS) [12]. Installing a dedicated RFQcb would decouple the CRIS beamline from the rest of ISOLDE; ions injected into the trap from ISOLDE and from CRISIS would have the same properties when they reach the CRIS interaction region. This would allow for continual optimisation of trapping parameters and efficiency with stable isotopes, improving beam quality and transport efficiency for radioactive beam experiments. Frequent reference measurements,

*christopher.ricketts@manchester.ac.uk performed by extracting a stable reference isotope from ISOLDE, are required during experiments on radioactive atoms to keep track of slow drifts in wavenumber readout. Switching masses on the ISOLDE separator for this purpose is time consuming (approximately 6 minutes per mass change); during recent CRIS campaigns, between $5 \%$ and $10 \%$ of the total beamtime was spent cycling the magnets. In addition, more than $33 \%$ of the remaining time was spent adjusting bending and focussing electric potentials to maximise ion beam transmission through CRIS A dedicated trap would allow CRISIS to be used for the reference measurements, and would almost entirely eliminate the beam transport optimisation required at the start of radioactive beam experiments. This period is when the yield of radioactive cases is at its maximum and additional data collection at this point would have a disproportionate effect on the overall signal significance. As the majority of background counts at CRIS scale with the beam current, for example non-resonant laser ionisation of isobaric contamination, the total background observed is proportional to the interaction region atom throughput, hence the signal significance scales with the product of the beam intensity, measurement time and total efficiency.

\section{Prototype RFQcb overview}

Anticipating the available lab space, the current prototype design uses a short trapping region, $20 \mathrm{~cm}$ in length, with four cylindrical rods at $5.3 \mathrm{~mm}$ internal radius. Sine or square wave $\mathrm{RF}$ driven at frequencies of the order of $\mathrm{MHz}$ will be applied to the rods to trap the ions radially, while helium buffer gas (at pressure in the range $10^{-3}$ to $10^{-1}$ mbar) will be used to reduce the amplitude of the radial oscillation. The ions will be trapped longitudinally using DC potentials applied to four printed circuit boards inserted between the rods. The rods and PCBs are mounted on two 3D printed endcap pieces. 

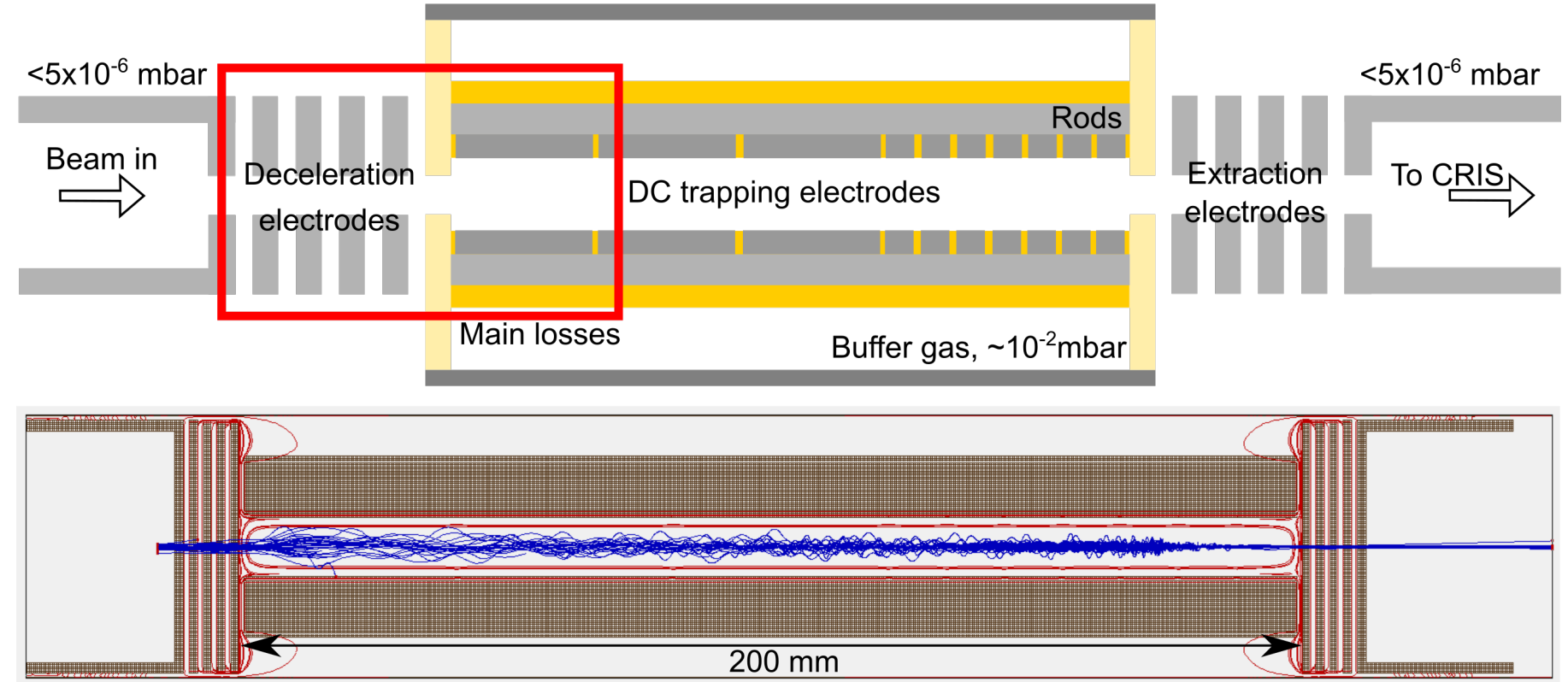

Figure 1: The current linear RFQ design. Upper - schematic of the trap design including the buffer gas containment cylinder (dark grey) around the trapping region, the 3D printed components (light yellow) and the PCB electrodes (dark yellow). 70\% of the losses occur in the region indicated by the red box. Lower - SIMION simulation showing electric potential contours (red) and $A=70$ ion trajectories starting at the beam waist, at 0.034 mbar buffer gas pressure (blue). The ions travel from left to right. Losses are minimal during extraction.

We have demonstrated that polylactic acid biopolymer (PLA) used in $3 \mathrm{D}$ printers is vacuum compatible. Figure 3 shows the pressure over time in a 3-way T-piece vacuum chamber with $440 \mathrm{~L} \mathrm{~s}^{-1}$ pumping capacity. As the figure shows, when a $20 \mathrm{~mm}$ side length cube of $3 \mathrm{D}$ printed PLA was placed in the vacuum chamber, the pressure decreased to $2 \times 10^{-7} \mathrm{mbar}$ after 5.5 hours of pumping, equal to the pressure reached by the empty chamber within the same pumping time. This pressure is compatible with the vacuum requirements of the RFQcb. Residual gas analysis was performed at $3.9 \times 10^{-8}$ mbar with the PLA sample present and at $6.7 \times 10^{-8}$ mbar when the chamber was empty. No significant change was seen in the composition of the gas after adding the sample, indicating that outgassing of the PLA is not significant at these pressures. The RGA spectra are shown in Figure 4. Flow rate calculations indicate the $4 \mathrm{~mm}$ apertures on either side of the trapping region will provide the required differential pumping to reach pressures below $5 \times 10^{-6}$ mbar outside the trap with two stages of differential pumping and up to 0.1 mbar internal buffer gas pressure.

The entire trap is biased to a potential slightly below $30 \mathrm{kV}$ to decelerate the incoming beam from $30 \mathrm{keV}$ to approximately $100 \mathrm{eV}$. At $30 \mathrm{keV}$, the Doppler broadening of the resonant excitation lineshape is comparable to the natural linewidth of many resonant transitions and a larger platform operating voltage is not required. SIMION simulations, shown in the lower part of Figure 1, have indicated that more than $70 \%$ of the ion beam losses occur during the injection-deceleration phase due to the narrow input aperture required for differential pumping and collisions with residual buffer gas. To mitigate these losses, a se- ries of PCB ring electrodes were introduced into the model to produce a longitudinal potential structure of the form $V(z)=$ $V_{0}\left(1-e^{-\alpha z}\right)$. This produces a transverse focussing effect with a focal point at a position proportional to $1 / \alpha$ [13]. The results of the simulations are shown in Table 1 and an output emittance plot for $A=70$ is shown in Figure 2. These used an input $30 \mathrm{keV}$ focussed beam with $20 \pi \mathrm{mm}$ mrad transverse emittance and a Gaussian radial profile with $0.5 \mathrm{~mm}$ standard deviation. The ions were released from the simulated trap after $2 \mathrm{~ms}$. The transmission efficiency at $A=70$ was $54 \%$ and the final simulated emittance of the cooled beam at this mass was $1.5 \pi \mathrm{mm}$ mrad.

\section{Outlook}

The benefits of installing an independent gas-filled linear Paul trap at the CRIS beamline have been explained and the design outlined is under development. Simulations have indicated the feasibility of a short trap design and have informed possible design improvements. The design offers a cost effective and high efficiency method to trap ions for radioactive beam studies. This work also illustrates the possible uses of 3D printed parts in vacuum, enabling cheaper and faster methods for design prototyping.

\section{Acknowledgements}

The authors would like to thank Colin Reed and Andrew McFarlane for preparing the PLA printed component and vacuum testing samples. This work was supported by the ERC 


\begin{tabular}{ccccc}
\hline \hline Mass, A & Pressure / mbar & Transmission probability / \% & Acceptance $/ \pi \mathrm{mm}$ mrad & Output emittance $/ \pi \mathrm{mm}$ mrad \\
\hline 12 & 0.012 & 11 & 10 & 16 \\
\hline 70 & 0.034 & 54 & 20 & 1.5 \\
\hline
\end{tabular}

Table 1: Simulated RFQ trapping parameters with total ion transmission probabilities, the input beam acceptance and output beam emittance after reacceleration at optimised buffer gas pressures. A $30 \mathrm{keV}$ focussed input beam with $20 \pi \mathrm{mm}$ mrad transverse emittance and a Gaussian radial profile with $0.5 \mathrm{~mm}$ standard deviation was used. The ions were released after $2 \mathrm{~ms}$.

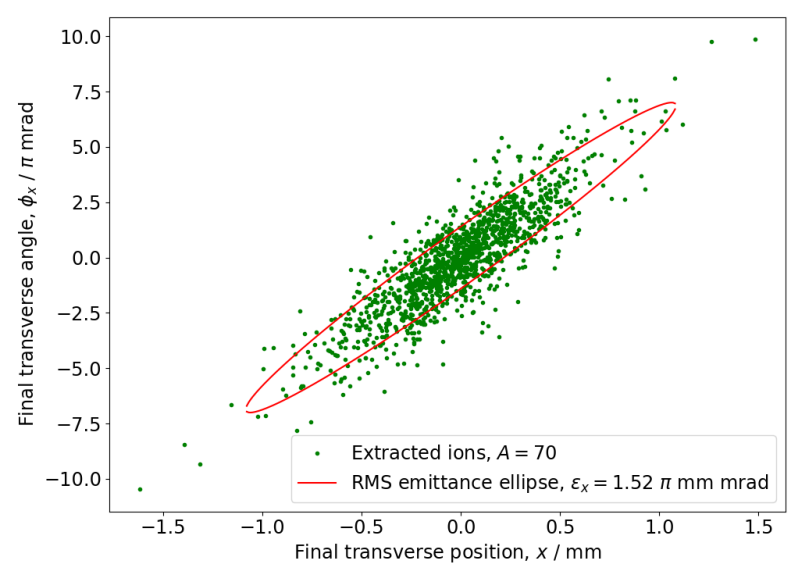

Figure 2: A plot showing the displacement along the transverse $x$-axis vs the velocity angle relative to the beam axis in the $x z$-plane, where $z$ is aligned along the beam axis, of the transmitted $A=70$ ions in a SIMION simulation of the RFQcb (green points). The ellipse showing the RMS transverse emittance in $x$ is also included (red line).

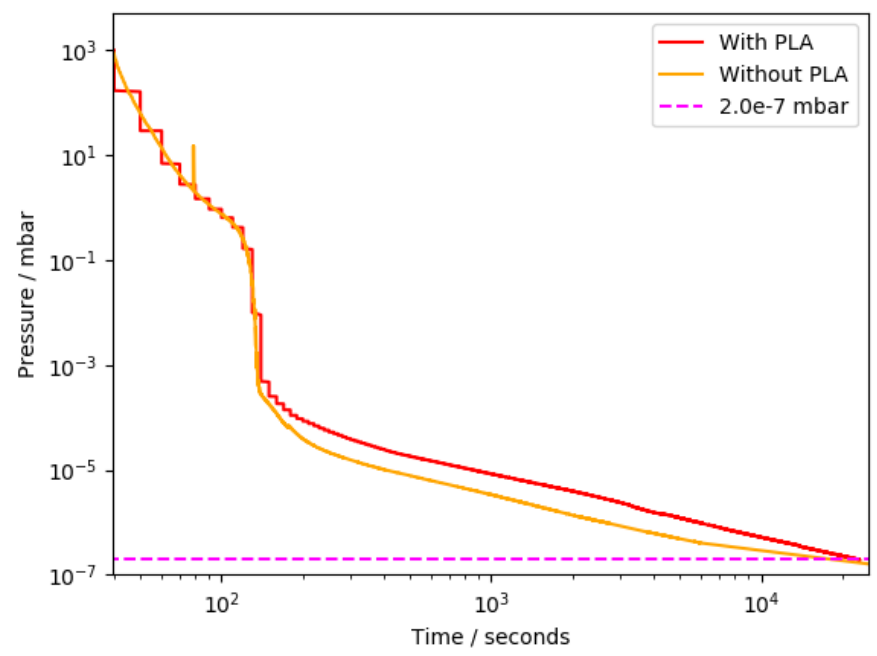

Figure 3: Pump down curves for an empty chamber (yellow) and for the same chamber with a $20 \mathrm{~mm}$ side length cube of PLA (orange). The chamber containing the PLA sample reached a pressure equal to that of the empty chamber, $2 \times 10^{-7}$ mbar, after 5.5 hours of pumping, indicated by the pink dashed line. Features in both curves below the order of $2 \times 10^{2} \mathrm{~s}$ are related to different pumping stages turning on.

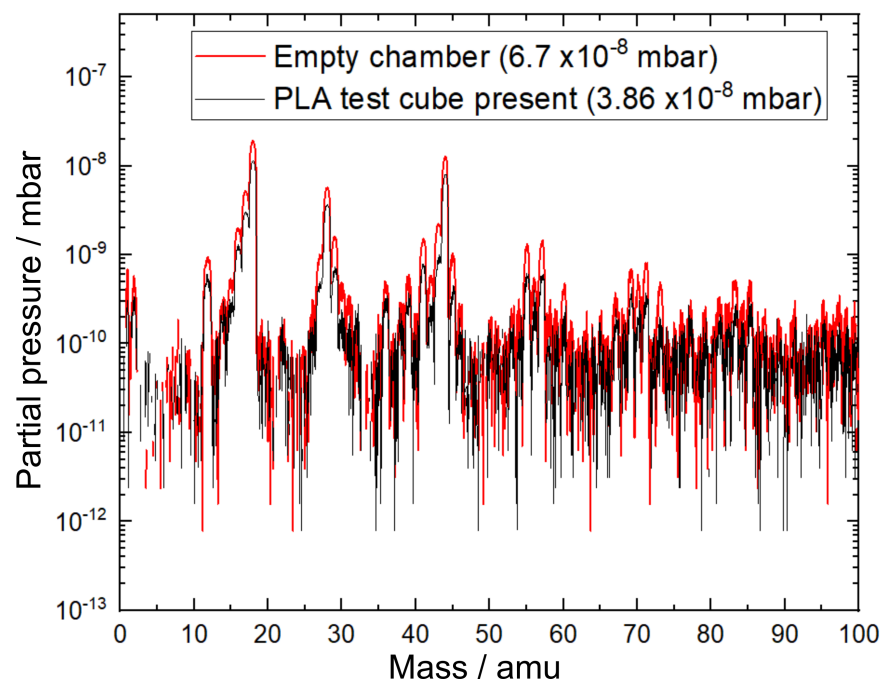

Figure 4: A plot showing the partial pressure at each mass detected by a residual gas analyser of the empty vacuum chamber at $6.7 \times 10^{-8} \mathrm{mbar}$ (red) and the vacuum chamber containing the PLA test cube at $3.9 \times 10^{-8}$ mbar (black). No significant change in the RGA spectrum is seen after adding the PLA sample, indicating that outgassing from the sample is not significant at these pressures.

Consolidator Grant No. 648381 and the Science and Technology Facilities Council Grants ST/P004423/1 and ST/S002316/1.

\section{References}

[1] A. Nieminen, et al. Phys. Rev. Lett., 88:094801, 2002.

[2] F. Herfurth, et al. Nuclear Instruments and Methods in Physics Research Section A: Accelerators, Spectrometers, Detectors and Associated Equipment, 469(2):254 , 2001. ISSN 0168-9002.

[3] T. Brunner, et al. Nuclear Instruments and Methods in Physics Research Section A: Accelerators, Spectrometers, Detectors and Associated Equipment, 676:32, 2012. ISSN 0168-9002.

[4] Y. A. Kudriavtsev et al. Applied Physics B, 29(3):219, 1982. ISSN 14320649.

[5] K. T. Flanagan, et al. Phys. Rev. Lett., 111:212501, 2013.

[6] C. Schulz, et al. Journal of Physics B: Atomic, Molecular and Optical Physics, 24(22):4831, 1991.

[7] R. P. de Groote, et al. Phys. Rev. Lett., 115:132501, 2015.

[8] Á. Koszorús, et al. In preparation.

[9] A. Vernon, et al. In preparation.

[10] M. J. G. Borge et al. Journal of Physics G: Nuclear and Particle Physics, 45(1):010301, 2018.

[11] E. Mané, et al. The European Physical Journal A, 42(3):503, 2009. ISSN 1434-601X.

[12] R. F. Garcia Ruiz, et al. Phys. Rev. X, 8:041005, 2018.

[13] R. F. Garcia Ruiz et al. Collinear Laser Spectroscopy on exotic Ca isotopes towards new magic numbers $N=32$ and $N=34,2015$. Presented 01 Oct 2015. 